\title{
Research Square \\ Prognostic factors for the 6-year cognitive impairment in China: results from the CLHLS cohort
}

\author{
Mingyue Hu \\ Xinyin Wu \\ Central South University \\ Xinhui Shu \\ Henan Cancer Hospital \\ Hengyu Hu \\ Central South University \\ Hui Feng (D 19174159734@163.com ) \\ https://orcid.org/0000-0001-6930-4780
}

Central South University Xiangya School of Medicine

\section{Research article}

Keywords: prognostic factors, cognitive impairment, the elderly, longitudinal study.

Posted Date: November 16th, 2019

DOI: https://doi.org/10.21203/rs.2.17402/v1

License: (c) (1) This work is licensed under a Creative Commons Attribution 4.0 International License. Read Full License 


\section{Abstract}

There is merely study from China using nationally representative longitudinal dataset to assess the prognostic factors for the progression of normal cognition to cognitive impairment (Cl).Aim This study will identify the best subset of 6-year $\mathrm{Cl}$ predictors.Design and setting 11,781 participants 60 years old or older from Chinese Longitudinal Healthy Longevity Survey (CLHLS, 2008-09 wave) were included at baseline for six-year follow-up. Of these individuals, 4,727 participants were eligible for final analysis. Univariate and binary logistic regression analysis was used to identify the significantly related predictors for incident $\mathrm{Cl}$. Subsequently, nomogram models were established to rank and find prognostic factors.Results A overall prevalence of 6-year $\mathrm{Cl}$ was $17.4 \%$, and 60-70, 70-80, 81-90, 91- age groups were $5.4 \%, 9.5 \%, 26.1 \%$ and $50.3 \%$, respectively. In general, the activities of daily living and baseline cognition were valuable prognostic factors to predict $\mathrm{Cl}$, with the exception of unmodifiable factors. Age subgroup analysis showed that, among 60-70 old age group, cardiovascular diseases were valuable prognostic factors; among over 70-year old age groups, baseline cognitive function could be a valuable addition to $\mathrm{Cl}$ prediction models. Conclusions Brief baseline cognitive testing and functional status were important valuable prognostic factors for $\mathrm{Cl}$.

\section{Background}

According to World Alzheimer Report 2015, china is the largest population of patients with dementia in the world ( 9.5 million), followed by USA ( 4.2 million)[1]. Identifying predictors of cognitive impairment (Cl) is important as it could reduce the incidence of $\mathrm{Cl}$ or dementia or substantially delay its onset. Delaying the onset of dementia by just one year is likely to reduce its prevalence by $11 \%$ by 2050 , while delaying it by five years could halve the number of people living with dementia by 2050[2].

There are several studies with robust evidence examining the modifiable prognostic risk factors for $\mathrm{CI}[2-$ 4]. A population-based perspective research from lancet commission[2] identified nine potentially prognostic factors of $\mathrm{Cl}$, including low educational level in childhood, hearing loss, hypertension, obesity, smoking, depression, physical inactivity, social isolation, and diabetes. A systematic review from the Alzheimer's Association[3] demonstrated that undernutrition was also risk factor of cognitive decline. Overall, the results were controversial and it might due to cultural difference around the world. The World Health Organization advocated that every country should routinely collect a core set of dementia indicators through their national health and social information systems[5]. However, there is little study examining risk prognostic factors of $\mathrm{Cl}$ from China. Therefore, this study will use Chinese Longitudinal Healthy Longevity Survey (CLHLS) database to explore prognostic factors for $\mathrm{Cl}$ and develop nomogram to predict 6-year $\mathrm{Cl}$.

\section{Methods}

Study population 
The data used in this study are from the Chinese Longitudinal Healthy Longevity Survey (CLHLS), an ongoing longitudinal survey launched in 1998 with follow-up surveys implemented in 2000, 2002, 2005, 2008-09, 2011-12 and 2014, respectively[6]. CLHLS is representative of the older population in China because the respondents are randomly sampled and the sampling frame covers about $85 \%$ of the total population of China[7].

Analytic sample

A total of 16,954 respondents at baseline were initially included in 2008-09. For this analysis, we excluded participants who were: 1) younger than 60 years old (114 participants); 2) were institutionalized (308 participants); 3) suffered mental diseases, including dementia (166) and Parkinson's disease (59); 4) suffered severe disease, like cancer (71); and 5) had cognitive impairment with MMSE scores lower than 18 points (4455 participants). Among the remaining participants, in 2011-12 wave, 1910 participants were lost to follow-up and 2879 died. In 2014 wave, 488 participants were lost to follow-up and 1777 died. The final dataset contained 4727 participants. Those who were excluded from analyses due to lost or death were on average older and had a lower baseline cognition score.

\section{Variables}

Sociodemographic information Sociodemographic factors were collected during the standardized interview. These included age (60-70, 71-80, 81-90 and 91-), sex, ethnicity (Han vs non-Han), marriage (partnered vs not partnered), economy (rich, fair and poor), co-residence status (living with other household member vs alone) and education (illiterate, 1-5 years, and $\geq 6$ years). Economy information was derived from asking participants 'how do you rate your economic status.'

Lifestyle behaviors Lifestyle behaviors contained smoking (current smoker, former smokers and nonsmoker), drinking (current drinker, former drinker and non-drinker). Exercise information were determined by the response to the question "Do you exercise or not at present" and responses were yes or no. Fruit and vegetable information were collapsed into three categories (often, occasionally and rarely) by asking the question "how often do you eat fresh fruit?". Social participation information was derived by the question 'do you take part in some social activities at present?' and was collapsed into four groups (several times a week, several times a month and rarely).

Psychological factors Self-rating health were combined into three groups (good, fair and bad) by the question "how do you rate your health." Loneliness and anxiety were determined by the questions "Do you always feel anxious and lonely" and responses were collapsed into four groups (often, sometimes, seldom and never).

Clinical diagnosis Hypertension, diabetes, heart diseases, stroke/cardiovascular diseases were determined by asking participants 'if these diseases were diagnosed by hospital?'

Anthropometric factors Body mass index (BMI) was calculated as the ratio of weight to squared height $(\mathrm{kg} / \mathrm{m} 2)$. Blood pressure (BP) was measured twice and the average systolic and diastolic BP was used, 
but if there was only one record of blood pressure, it then was used instead of the average. Functional impairment at baseline were measured by the Katz index of independence in activities of daily living (ADLs). Functional impairment was defined as having difficulty in performing at least one of the six ADLs.

Cognitive impairment Cognitive function was assessed using the Chinese version Mini-Mental State Examination (MMSE). It consists of 24 items within 6 dimensions: 5 items for Orientation, 3 for Registration, 1 for Naming, 5 for Attention and Calculation, 3 for Recall and 7 for Language. The total score of the Chinese MMSE ranges from 0 to 30 points; higher scores indicate better cognitive levels. The Chinese MMSE has been validated among Chinese elderly. Cognitive impairment was defined as a CMMSE score below 18 points as previously validated[8].

\section{Statistical analysis}

We used categorical patient characteristics such as the age by MMSE using the chi-square test, or, if expected cell counts were less than 5 , continuity correction was applied. We used Wilcoxon tests to compare two groups on $\mathrm{BMI}$, blood pressure and heart rate as all the test of normality did not show normal distribution ( $p$ for Kolmogorov-Smirnov test $<0.05$ ). These tests were all 2 sided.

We used binary logistic regression analysis was used to explore the association between variables and $\mathrm{Cl}$. Moreover, we developed the nomogram model via R package regplot using the result of the binary logistic regression model to show the odds ratio of each predictor and to assess the probability of $\mathrm{Cl}$.

Some covariates including education (7 missing), economy (6 missing), smoking (4 missing), drinking (4 missing), blood pressure (115 missing), heart rate (119 missing), BMI (18 missing), self-rated anxiety (32 missing) and self-rated loneliness (24 missing), had missing values. However, the proportion of missing values was $<5 \%$ for all variables. Thus, we performed imputations, using multivariable regression methods via R package mice. All statistical analysis was performed with free open-source R statistical software (www.r-project.org).

\section{Results}

The baseline information was presented in Table 1. For binary and categorical variables, the number and percentage of participants in each category were represented. For continuous variables, the median $\left(25^{\text {th }}\right.$ and $75^{\text {th }}$ quantiles) were reported. A total number of participants who were cognitively normal at baseline and who processed to $\mathrm{Cl}$ was 821 (17.4\%). The baseline mean age of participants was $78.01,48.7 \%$ was male, $93.5 \%$ was Han and $50 \%$ were illiterate.

Univariate analysis showed that age, sex, education, marriage, smoking, drinking, fruit, vegetable, exercise, self-rated anxiety, self-rated loneliness, BMI, ADLs and baseline MMSE were significantly associated with incident $\mathrm{Cl}(\mathrm{p}<0.05)$. (see Table 1$)$.

Table 1. Baseline characteristics of the study population by CI. 


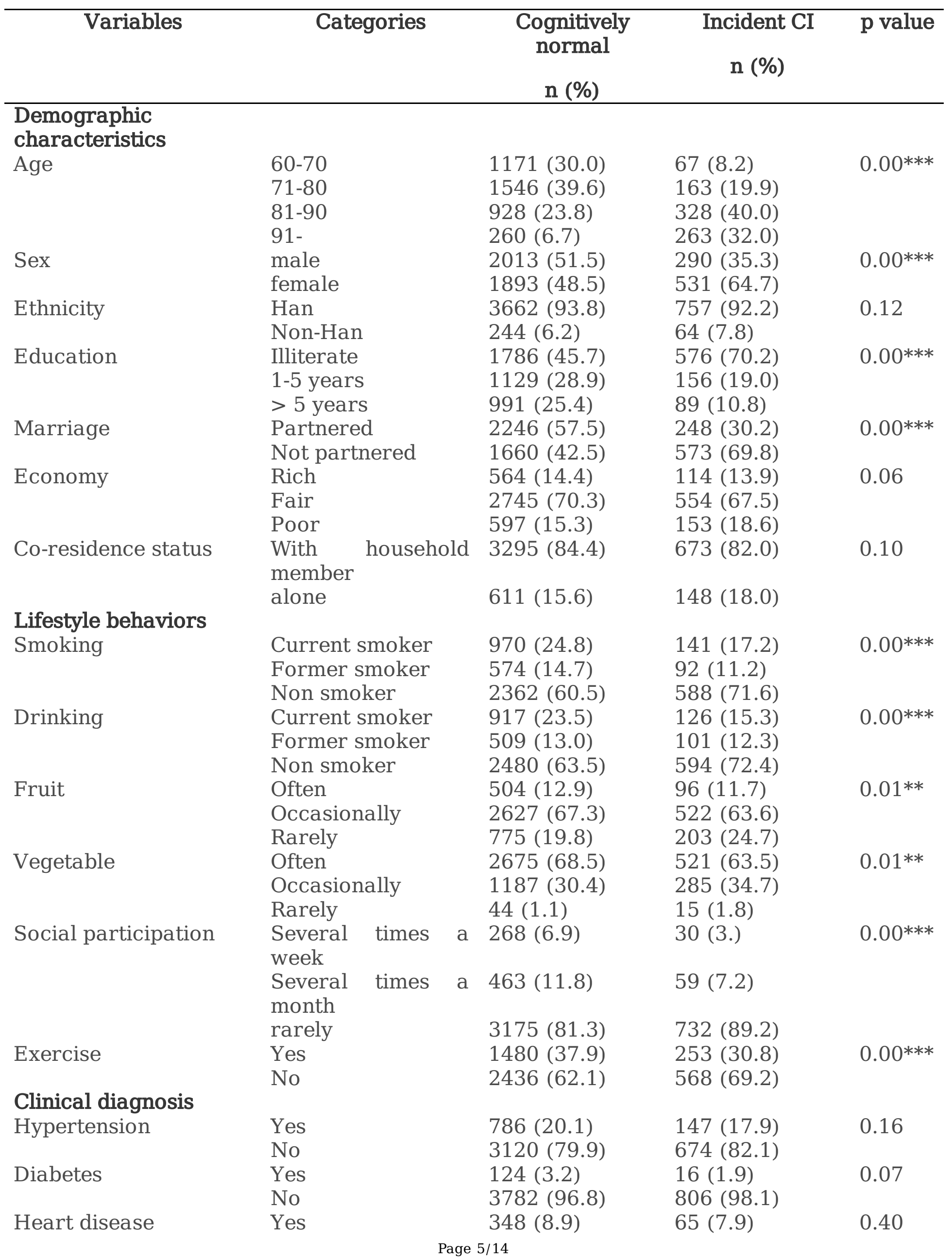




\begin{tabular}{|c|c|c|c|c|}
\hline & No & $3558(91.1)$ & $756(92.1)$ & \multirow{3}{*}{0.48} \\
\hline \multirow[t]{2}{*}{ Stroke or $\mathrm{CVD}^{1}$} & Yes & $184(4.7)$ & $44(5.4)$ & \\
\hline & No & $3722(95.3)$ & $777(94.6)$ & \\
\hline \multicolumn{5}{|l|}{ Psychological factors } \\
\hline \multirow{3}{*}{ Self-rated health } & Good & 2177 (55.7) & $437(53.2)$ & \multirow[t]{3}{*}{0.32} \\
\hline & Fair & $1234(31.6)$ & $267(32.5)$ & \\
\hline & Bad & $495(12.7)$ & $117(14.3)$ & \\
\hline \multirow[t]{4}{*}{ Self-rated anxiety } & Often & $208(5.4)$ & $47(5.8)$ & \multirow[t]{4}{*}{$0.01 * *$} \\
\hline & Sometimes & 718 (18.5) & $182(22.4)$ & \\
\hline & Seldom & $1283(33.0)$ & $283(34.9)$ & \\
\hline & never & $1675(43.1)$ & $299(36.9)$ & \\
\hline \multirow[t]{4}{*}{ Self-rated loneliness } & Often & $235(6.0)$ & $67(8.2)$ & \multirow[t]{4}{*}{$0.00 * * *$} \\
\hline & Sometimes & $679(17.5)$ & $188(23.1)$ & \\
\hline & Seldom & $1173(30.2)$ & $269(33.0)$ & \\
\hline & never & $1801(46.3)$ & $291(35.7)$ & \\
\hline \multicolumn{5}{|l|}{$\begin{array}{l}\text { Anthropometric } \\
\text { factors }\end{array}$} \\
\hline $\mathrm{BMI}^{2}$ & & $\begin{array}{l}20.9(18.9, \\
23.4)\end{array}$ & $\begin{array}{l}20.1 \text { (17.9, } \\
22.5)\end{array}$ & $0.00 * * *$ \\
\hline $\mathrm{SBP}^{3}$ & & $\begin{array}{l}134.0(123.0, \\
149.5)\end{array}$ & $\begin{array}{l}133.5(122.5, \\
148.5)\end{array}$ & 0.78 \\
\hline $\mathrm{DBP}^{4}$ & & $\begin{array}{l}79.0(72.0, \\
85.5)\end{array}$ & $\begin{array}{l}79.0(72.0, \\
85.5)\end{array}$ & 0.82 \\
\hline Heart rate & & $\begin{array}{l}72.5(68.0, \\
78.5)\end{array}$ & $\begin{array}{l}73.0(68.5, \\
78.0)\end{array}$ & 0.28 \\
\hline $\mathrm{ADLs}^{5}$ & Impairment & $94(2.4)$ & $67(8.2)$ & $0.00 * * *$ \\
\hline & Non-impairment & $3812(97.6)$ & $754(91.8)$ & $\cap \cap \cap *$ \\
\hline Baseline MMSE $^{6}$ & & $29(26,30)$ & $27(23,30)$ & $0.00^{*}$ \\
\hline
\end{tabular}

$* \mathrm{p}<0.05 ;{ }^{* *} \mathrm{p}<0.01 ; * * \mathrm{p}<0.001$.

Abbreviation: ${ }^{1}$ CVD cardiovascular disease; ${ }^{2}$ BMI: body mass index; ${ }^{3}$ SBP: systolic blood pressure; ${ }^{4}$ DBP: diastolic blood pressure; ${ }^{5}$ ADLs: Katz activities of daily living; ${ }^{6}$ MMSE: Mini-mental State Examination.

Binary logistic regression analysis

Seven predictors were included in the binary logistic regression model, but only 5 predictors were significantly related to incident $\mathrm{Cl}$, including age, education, marriage, ADLs and baseline MMSE. (see table S1)

Nomogram predicting the incident $\mathrm{Cl}$ among individuals age 60 and older

According to the result of the binary logistic regression, we used 5 variables, including age, education, marriage, ADLs and baseline MMSE as predictors to develop a nomogram model. 
Nomogram (Figure 2) was used: 1 ) to observe the distribution of the participants in different variables. Precisely, for category variables, box size represented the proportion of the sample. For instance, as to ADLs, most of the participants were being 'not impairment' group as the box size was obviously bigger than 'impairment' group. For continuous variables, density exhibited the distribution of the sample. For example, as to baseline MMSE, most of individuals were scored over $25 ; 2$ ) to rank predictors in terms of 6 -year risk of incident $\mathrm{Cl}$. One hundred points were assigned to the most dangerous predictor (age $>90$ in this study), and the other predictors were given corresponding points based on weight. and 3) to estimate the cumulative effect of five predictors as follows. For instance, a sum of the points (red mark in Figure 2) for a subject of baseline MMSE score of 25, ADLs of not impairment, marriage of not partnered, education of illiterate and age of 81-90 equals a total of score of 124 and probability of incident $\mathrm{Cl}$ was $33.2 \%$.

\section{Subgroup analysis based on age groups}

As age was the most important predictor of incident, so we then explored the predictors in different age groups. Firstly, in four age groups, univariate analysis was used and variables with a $p$ value less than 0.05 was entered in binary logistic regression analysis, respectively. Then, statistically significant predictors of logistic regression analysis were utilized to make nomogram.

\section{Nomogram predicting the incident $\mathrm{Cl}$ among individuals aged 60-70}

The results of univariate and binary regression analysis of 60-70 age group were shown in table $S 2 \& 3$. Figure 3 showed that, among 60-70 age group, DBP ranged from about 50 to 110 and the most was 80 , and most participants were not diagnosed as stroke or CVD. These two predictors were significantly associated with $\mathrm{Cl}$. Participants with a DBP of 80 and no stroke/CVD had a probability of $4.6 \%$ having incident $\mathrm{Cl}$. 『see Figure S3区

\section{Nomogram predicting the incident $\mathrm{Cl}$ among individuals aged 71-80}

The results of univariate and binary regression analysis of 71-80 age group were shown in table S4\&5. Figure 4 showed that, among 71-80 age group, most individuals had baseline MMSE points of over 24, and number of participants in education category were evenly distributed. These two predictors were significantly associated with $\mathrm{Cl}$ in 71 to 80 age group. Participants with a baseline MMSE of 24 and education of illiterate had a probability of $15.0 \%$ having incident $\mathrm{Cl}$. (see Figure S4)

\section{Nomogram predicting the incident $\mathrm{Cl}$ among individuals aged 81-90}

The results of univariate and binary regression analysis of 81-90 age group were shown in table S6\&7. Figure 5 showed that, among 81-90 age group, baseline MMSE were mostly over 24, the SBP ranged from 100 to 200 , and number of participants in marriage category were evenly distributed. These three predictors were significantly associated with $\mathrm{Cl}$ in 81 to 90 age group. Participants with a baseline MMSE of 24 , SBP of 130 and marriage of not partnered had a probability of $34.5 \%$ having incident $\mathrm{Cl}$. (see Figure S5) 
The results of univariate and binary regression analysis of 90 - age group were shown in table $8 \& 9$. Figure 6 showed that, among 91- age group, baseline MMSE points and sex were evenly distributed, and most participants in education category were illiterate. These three predictors were significantly associated with $\mathrm{Cl}$ in 91- age group. Participants with a baseline MMSE of 24, education of illiterate and female had a probability of $61.1 \%$ having incident $\mathrm{Cl}$. (see Figure S6)

\section{Validation of the Nomogram Model}

C-indexes were calculated for each of the adjusted models to illustrate the predictive ability of the independent variables. The $\mathrm{C}$-index is a value that indicates the concordance level between observed and expected ordering of the data, and represents the percentage of concordance of all pairs of data with different outcome values, with concordance defined as occurring within a pair when the observation with the higher outcome value also has the higher predicted probability of the outcome. The C-index were $0.775,0.653,0.641,0.617$ and 0.661 .

\section{Discussion}

Our Results showed that modifiable indicators (baseline cognitive function and ADLs), and nonmodifiable factors (age, education and marriage) were strong predictors forecasting $\mathrm{Cl}$. Recent metaanalysis[9] of 61 cohort studies regarding the predictive model for risk of dementia summarized common predictive factors used in included studies including demographic factors (age, education and sex), cognitive function, health and lifestyles and genetics. In consistent with this study, we found modifiable factor of cognitive function was valuable to predict dementia but our study was not able to include genetics information. One recent study[10] using UK Biobank database involving almost 500,000 people explored whether cognitive function could predict dementia and to which discriminative accuracy it could contribute in terms of distinguishing dementia from non-dementia people. This study confirmed that brief cognitive testing could be a valuable addition to dementia prediction models.

As for the predictable strengthen of functional ability for $\mathrm{Cl}$, a meta-analysis[11] of 36 cohort researches demonstrated that physical function at baseline significantly related to longitudinal changes in cognitive function. Decreases in grip strength was sensitive to cognitive function decline and changes in walking speed was strongly related fluid cognition like memory and executive function[11]. Consistently, gait speed and strength were associated with cognitive function, especially the executive function[12] and daily walking speed significantly related to executive function[13]. Additionally, there was also study suggested that cognitive and physical function influence each other's development[14]. The underlying why physical could forecast $\mathrm{Cl}$ might be that physical function like walking speed requires motor coordination and balance that are regulated by cerebellum[15]. Furthermore, the cerebellum connected to cortical associative areas supporting higher mental function like the prefrontal cortex, which regulates several aspects of fluid cognitive skills. Moreover, gait speed is correlated to brain white-matter integrity that plays important role in the connecting brain systems[16, 17]. Biological mechanism could also 
explain the role of physical ability in cognitive function decline. Physical function upregulates brainderived neurotrophic factor, leading to stimulation of the dentate gyrus in the hippocampus that is the important region of cognition of memory[18, 19].

Our study found higher DBP was significantly associated with lower cognitive scores in younger elders but lower SBP significantly related to cognitive decline in older elders. The association between BP and cognition is controversial. A systematic review demenstrated positive, negative and $\mathrm{J}$ - and $\mathrm{U}$-shaped associations between BP and cognition[20]. Our study was consistent with previous study involving magnetic resonance imaging (MRI) detecting the relationship between $\mathrm{BP}$ and cognition, with reduced hippocampal volume and higher white matter intensities (WMHs) indicating Cl pathology. Result showed that higher DBP predicted WMHs burden and smaller hippocampi whereas increased SBP predicted larger hippocampi[21]. One possible mechanism underlying this finding is that increased DBP is a risk factor or marker of early cognitive impairment pathology in this ageing sample, whereas increased SBP protects hippocampal volume from the deleterious effects of DBP through the maintenance of blood supply[22]. Overall, in our Cl-free cohort, DBP is associated with 6-year $\mathrm{Cl}$ or accelerate the development of preexisting pathologies.

\section{Limitations}

Our study had several limitations. Firstly, cognitive impairment was measured by Chinese version MMSE and this study did not adjust for education. This was due to cultural difference, as most of older people in China were illiterate in 20th century. However, this scale has reported high sensitivity and specificity to detect cognitive impairment. Secondly, missing data due to lost and deats from participants with lower cognitive ability and/or older age were likely to have reduced the sensitivity of the analysis due to selective attrition, but the effect size of participants with lower cognition scores who were more likely to lost $(O R=1.06)$ and died $(O R=1.17)$ were small. Thirdly, our study did not include genetics information that could add additional value to cognitive impairment prediction model. However, the baseline characteristics promised to be a noninvasive, convenient means of indicating cognitive impairment, and the baseline cognitive and ADL tests administered to participants were particularly brief. Therefore, further study should further include genetic information of Chinese people and examine their additional value to cognitive impairment prediction model. Fourthly, the measurement of functional ability was ADL, and indicators that were more objective such as walking speed and grip strength might be more brief and sensitive to cognitive impairment. Fifthly, we did not explore the effect of subdomains of MMSE such as memory on cognitive impairment. Many studies have demonstrated that tests of specific cognitive domains that were most predictive include episodic memory, executive function, verbal fluency, and processing speed. So, future studies could further deeply explore the effect of subdomains of cognition on future cognitive impairment among Chinese people and analyze the difference of results between China and other nations.

\section{Conclusions}


The overall prevalence of 6-year $\mathrm{Cl}$ was high in 91-age groups (50.3\%). The activities of daily living and baseline cognition were valuable prognostic factors to predict $\mathrm{Cl}$ across nearly all age groups. However, among 60-70 old age group, cardiovascular diseases were valuable prognostic factors.

\section{Abbreviations}

CLHLS: Chinese Longitudinal Healthy Longevity Survey; CVD: cardiovascular disease; BMI: body mass index; SBP: systolic blood pressure; DBP: diastolic blood pressure; ADLs: Katz activities of daily living; MMSE: Mini-mental State Examination.

\section{Declarations}

\section{Acknowledgements}

We thank all participants of the CLHLS study and all members of the CLHLS study team. The data used in this research were provided by the Chinese Longitudinal Healthy Longevity Survey (CLHLS) study, which was managed by the Center for Healthy Ageing and Development Studies, Peking University. The CLHLS was supported by funds from the US National Institute on Ageing (NIA), China Natural Science Foundation, China Social Science Foundation and United Nations Fund for Population Activities (UNFPA).

\section{Authors' contributions}

FH conceived and designed the study, and supervised the data analysis. HMY and HMY analysed the data and wrote the paper together. JW helped with revising the manuscript. WXY gave advice on statistical analysis. All authors have given final approval of the version to be published.

\section{Funding}

With the exception of funding of CLHLS, this research was funded by the Research and Development Program in Key areas of Hunan Province, China Hunan Provincial Science and Technology Department (grant number: 2019SK2143).

\section{Availability of data and materials}

The CLHLS dataset is publicly available. All data used in this study was stored at Peking university (http://opendata.pku.edu.cn/) and available upon request.

\section{Ethics approval and consent to participate}

Ethical approval and consent to participate The CLHLS study was approved by research ethics committees of Duke University and Peking University (IRB00001052-13074).

\section{Consent for publication}


Not applicable.

\section{Competing interests}

The authors declare that they have no competing interests.

\section{References}

1. Prince M, Wimo A, Guerchet M, Ali G, Wu Y, Prina M: World Alzheimer Report 2015: The Global Impact of Dementia: an Analysis of Prevalence, Incidence, Cost and Trends. Alzheimer's Disease International.

2. Frankish $\mathrm{H}$, Horton R: Prevention and management of dementia: a priority for public health. Lancet (London, England) 2017, 390(10113):2614-5.

3. Baumgart M, Snyder HM, Carrillo MC, Fazio S, Kim H, Johns H: Summary of the evidence on modifiable risk factors for cognitive decline and dementia: a population-based perspective. Alzheimer's \& Dementia 2015, 11(6):718-26.

4. Beydoun MA, Beydoun HA, Gamaldo AA, Teel A, Zonderman AB, Wang Y: Epidemiologic studies of modifiable factors associated with cognition and dementia: systematic review and meta-analysis. BMC public health 2014, 14(1):643.

5. Organization WH: Global action plan on the public health response to dementia 2017-2025. 2017.

6. The Chinese Longitudinal Healthy Longevity Study. https://sites.duke.edu/centerforaging/programs/chinese-longitudinal-healthy-longevity-survey-clhls/. Accessed 1 October 2019.

7. Zeng Y: Chinese longitudinal healthy longevity survey and some research findings. Geriatrics \& Gerontology International 2004, 4:S49-S52.

8. Zhang Z-X, Zahner GE, Roman GC, Liu X-H, Wu C-B, Hong Z, Hong X, Tang M-N, Zhou B, Qu Q-M: Socio-demographic variation of dementia subtypes in china: Methodology and results of a prevalence study in Beijing, Chengdu, Shanghai, and Xian. Neuroepidemiology 2006, 27(4):177-87.

9. Hou X-H, Yu J-T: Models for Predicting Risk of Dementia: A Systematic Review. Alzheimer's \& Dementia 2018, 14(7):P803.

10. Calvin CM, Wilkinson T, Starr JM, Sudlow C, Hagenaars SP, Harris SE, Schnier C, Davies G, FawnsRitchie C, Gale CR et al: Predicting incident dementia 3-8 years after brief cognitive tests in the UK Biobank prospective study of 500,000 people. Alzheimers Dement 2019.

11. Clouston SA, Brewster P, Kuh D, Richards M, Cooper R, Hardy R, Rubin MS, Hofer SM: The dynamic relationship between physical function and cognition in longitudinal aging cohorts. Epidemiologic reviews 2013, 35(1):33-50.

12. Rosano C, Simonsick EM, Harris TB, Kritchevsky SB, Brach J, Visser M, Yaffe K, Newman AB: Association between physical and cognitive function in healthy elderly: the health, aging and body composition study. Neuroepidemiology 2005, 24(1-2):8-14. 
13. Mielke MM, Roberts RO, Savica R, Cha R, Drubach DI, Christianson T, Pankratz VS, Geda YE, Machulda MM, Ivnik RJ: Assessing the temporal relationship between cognition and gait: slow gait predicts cognitive decline in the Mayo Clinic Study of Aging. Journals of Gerontology Series A: Biomedical Sciences and Medical Sciences 2012, 68(8):929-37.

14. Bishop NJ, Eggum-Wilkens ND, Haas SA, Kronenfeld JJ: Estimating the co-development of cognitive decline and physical mobility limitations in older US adults. Demography 2016, 53(2):337-64.

15. Baillieux H, De Smet HJ, Paquier PF, De Deyn PP, Mariën P: Cerebellar neurocognition: insights into the bottom of the brain. Clinical neurology and neurosurgery 2008, 110(8):763-73.

16. Starr JM, Leaper S, Murray AD, Lemmon H, Staff RT, Deary IJ, Whalley LJ: Brain white matter lesions detected by magnetic resosnance imaging are associated with balance and gait speed. Journal of Neurology, Neurosurgery \& Psychiatry 2003, 74(1):94-8.

17. Penke L, Maniega SM, Murray C, Gow AJ, Hernández MCV, Clayden JD, Starr JM, Wardlaw JM, Bastin ME, Deary IJ: A general factor of brain white matter integrity predicts information processing speed in healthy older people. Journal of Neuroscience 2010, 30(22):7569-74.

18. Van Praag H, Christie BR, Sejnowski TJ, Gage FH: Running enhances neurogenesis, learning, and long-term potentiation in mice. Proceedings of the National Academy of Sciences 1999, 96(23):13427-31.

19. Van Praag H, Kempermann G, Gage FH: Running increases cell proliferation and neurogenesis in the adult mouse dentate gyrus. Nature neuroscience 1999, 2(3):266.

20. J B, L K: Cognitive function and hypertension. Journal of Human Hypertension 2009, 23:86-96.

21. McNeil CJ, Myint PK, Sandu A-L, Potter JF, Staff R, Whalley LJ, Murray AD: Increased diastolic blood pressure is associated with MRI biomarkers of dementia-related brain pathology in normative ageing. Age and ageing 2017, 47(1):95-100.

22. Horsburgh K, Reimer MM, Holland P, Chen G, Scullion G, Fowler JH: Axon-glial disruption: the link between vascular disease and Alzheimer's disease? In.: Portland Press Limited; 2011.

\section{Additional File Legends}

Additional files: Table S1. Binary logistic regression analysis. Table S2. Baseline characteristics of the study population by $\mathrm{Cl}$ (60-70 age group). Table S3. Binary logistic regression analysis (60-70 age group). Table S4. Baseline characteristics of the study population by $\mathrm{Cl}$ (71-80 age group). Table S5. Binary logistic regression analysis (71-80 age group). Table $\mathbf{S 6}$. Baseline characteristics of the study population by $\mathrm{Cl}$ (81-90 age group). Table S7. Binary logistic regression analysis (81-90 age group). Table S8. Baseline characteristics of the study population by $\mathrm{Cl}$ (91-age group). Table S9. Binary logistic regression analysis (90-age group). Figure S3. Nomogram predicting the incident $\mathrm{Cl}$ among individuals aged 60-70. Figure S4. Nomogram predicting the incident $\mathrm{Cl}$ among individuals aged 71-80. Figure S5. Nomogram 
predicting the incident $\mathrm{Cl}$ among individuals aged 81-90. Figure S6. Nomogram predicting the incident $\mathrm{Cl}$ among individuals aged over 90 .

\section{Figures}

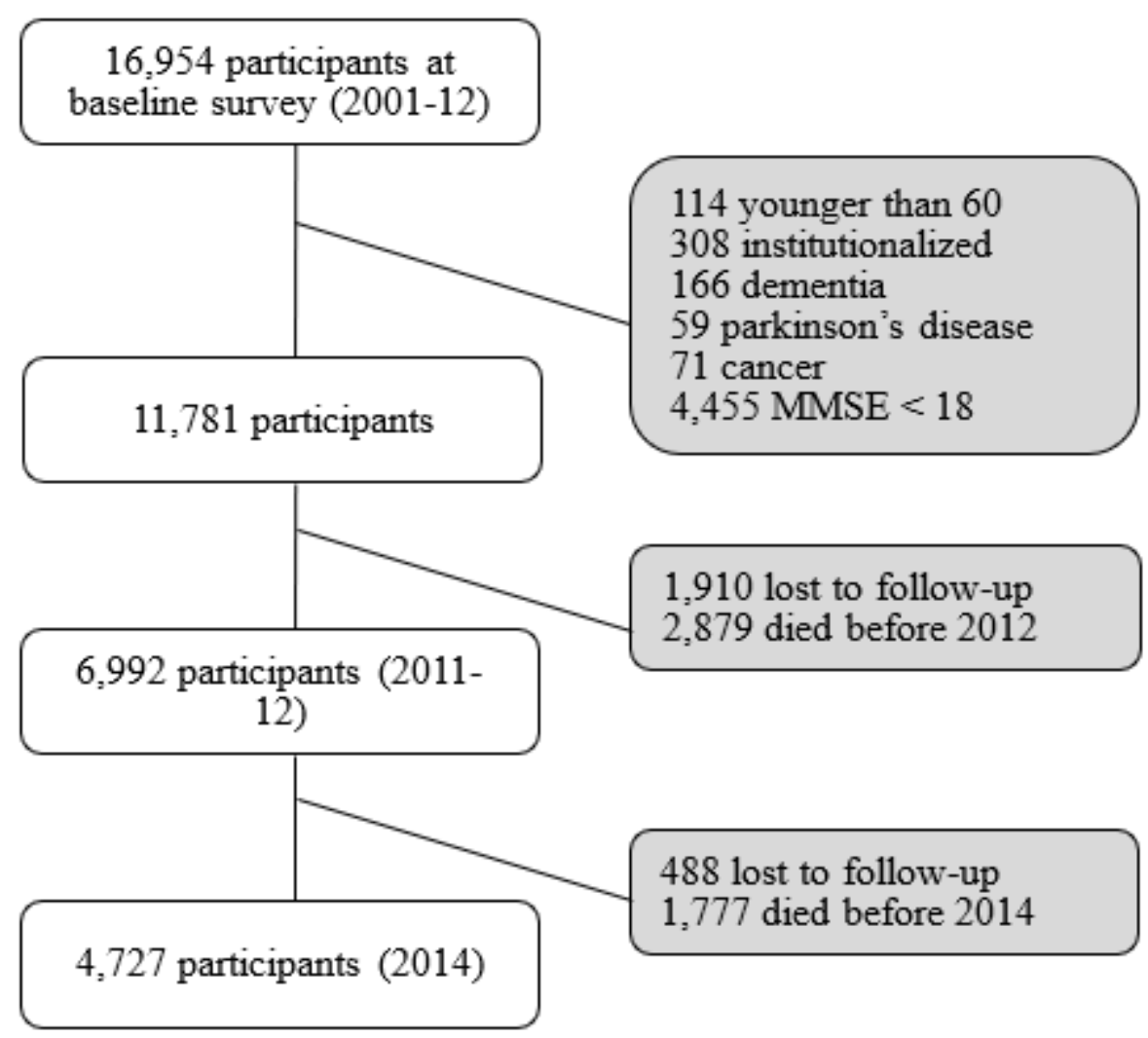

\section{Figure 1}

flow-chart of participant selection.






\section{Figure 2}

Nomogram ranking the predictors showing the cumulative effect of predictors. First, each variable is marked on the appropriate scale, and the number of points for each is derived from the 'Points' scale at the top of the nomogram. Then the points are totaled, and the total value is marked on the "Total Points" scale. Viewing down, one then derives the total score on the scale labeled ' $\mathrm{Pr}^{\prime}$ meaning means the probability of incident $\mathrm{Cl}$.

\section{Supplementary Files}

This is a list of supplementary files associated with this preprint. Click to download.

- Supplementarymaterial.pdf 